\title{
Editorial
}

\section{SIDA: Epidémiologie et prévention}

Pas de traitement efficace et un vaccin qui se fera attendre longtemps encore. Une maladie mortelle qui se propage dans le monde entier, voilà bien un problème de médecine sociale et préventive.

Et pourtant la gravité du problème est encore trop souvent méconnue. On ne met pas assez à profit les expériences faites dans d'autres domaines de la santé, ni les connaissances les plus récentes en matière de prévention pour mettre en cuvre une éducation sanitaire efficace. Pour l'heure, la propagation du HIV ne peut être enrayée que par un changement des comportements. On sait l'essentiel sur la façon d'influencer le comportement de l'homme et les raisons pour lesquelles il le modifie.

Les articles publiés dans ce numéro voudraient contribuer à orienter les esprits dans cette direction. Les auteurs ont essayé de tirer des données épidémiologiques les conclusions qui s'imposent sur le plan de la prévention, dans le milieu de la drogue (Uchtenhagen), celui de la prostitution (Piot) et celui des homosexuels (Samuel). Une attention toute particulière doit être vouée aux jeunes (Michaud), car ce groupe de la population est potentiellement le plus menacé. L'information de l'ensemble de la population (Somaini) joue cependant un rôle décisif, car, faute de cette information, la prévention spécifique aux différents groupes ne saurait être efficace. Les aspects éthiques (Bondolfi) jouent un rôle important dans la prévention du SIDA, car cette maladie touche à la morale et aux tabous. Le contrôle de l'efficacité de telles mesures est tout aussi important. Les articles sur l'évaluation (Lehmann, Mathey) traitent le sujet de façon détaillée.

On ne pourra atteindre les buts fixés, à savoir: empêcher de nouvelles infections et promouvoir la solidarité, que si nous réussissons à faire étroitement converger la prévention spécifique aux groupes, la prévention individuelle et les messages adressés à l'ensemble de la population.

Le problème de la drogue est typique à cet égard. Les mesures de lutte contre le SIDA n'auront d'impact sur ce groupe que si la toxicomanie est perçue comme un problème de notre société et que celle-ci, face à l'infection HIV, s'emploie à trouver une solution basée sur la solidarité.

Le SIDA est un problème qui relève de la médecine sociale et préventive. Nous pouvons cuvrer activement à sa solution, en mettant nos connaissances en pratique.

Bertino Somaini, Berne 


\section{Editorial}

\section{AIDS: Epidemiologie und Prävention}

Keine wirksame Therapie ist vorhanden, ein möglicher Impfstoff liegt noch in weiter Ferne. Die Epidemie einer tödlichen Krankheit, welche sich weltweit ausbreitet - das ist wahrlich ein Thema für die Sozial- und Präventivmedizin-sollte man meinen.

Doch wird leider noch allzuoft die tiefgreifende Problematik nicht erkannt. Die gemachten Erfahrungen aus anderen Gesundheitsbereichen und auch die neueren Erkenntnisse im Bereiche der Prävention werden für eine erfolgversprechende Gesundheitserziehung zuwenig berücksichtigt. Die HIV-Ausbreitung kann vorläufig nur mit einer Verhaltensänderung bekämpft werden. Wie menschliches Verhalten zu beeinflussen ist oder warum es sich ändert - darüber existieren wesentliche Erkenntnisse.

Die folgenden Beiträge möchten in dieser Richtung motivierend wirken. Es wurde versucht, aus den epidemiologischen Erkenntnissen die nötigen präventiven Schlussfolgerungen zu ziehen. So am Beispiel der Drogenabhängigen (Uchtenhagen), im Bereich der Prostitution (Piot) oder bei Homosexuellen (Samuel). Den Jugendlichen (Michaud) muss spezielle Aufmerksamkeit geschenkt werden, denn diese Gruppe ist potentiell stärker gefährdet. Allerdings spielt die Aufklärung in der Gesamtbevölkerung (Somaini) eine entscheidende Rolle, da ohne diese Massnahmen zielgruppenspezifische Prävention kaum wirkungsvoll sein kann. Die ethischen Aspekte (Bondolfi) spielen bei der AIDS-Prävention eine wichtige Rolle, da hier moralische Bereiche und Tabus betroffen sind. Wichtig bei allen solchen Massnahmen ist eine Erfolgskontrolle. Die Beiträge über die Evaluation (Lehmann, Mathey) gehen näher darauf ein.

Nur wenn es uns gelingt, die zielgruppenspezifische oder die Individualprävention eng mit den Botschaften an die Gesamtbevölkerung zu verknüpfen, wird es möglich sein, die gesteckten Ziele zu erreichen: Neuinfektion zu verhindern und die Solidarität zu fördern. Dies wird deutlich beim Drogenproblem. Nur wenn die Gesellschaft das Drogenproblem als Problem unserer Gesellschaft akzeptiert und im Lichte der HIV-Infektion versucht, solidarisch eine Lösung zu suchen, nur dann werden die AIDS-Bekämpfungsmassnahmen auch bei dieser Gruppe erfolgversprechend sein.

AIDS ist ein Thema für die Sozial- und Präventivmedizin. Wir alle können unsere Kenntnisse auf diesem Gebiet aktiv in die Tat umsetzen.

Bertino Somaini, Bern 